\title{
ADAPTING A GIS VERSION OF THE IRVINE-MINNESOTA INVENTORY (IMI) FOR BULGARIAN SETTINGS
}

\author{
Angel Dzhambov ${ }^{1}$, Donka Dimitrova ${ }^{2}$, Mariana Aleksandrova ${ }^{3}$ \\ ${ }^{1}$ Department of Hygiene and ecomedicine, Faculty of Public Health, \\ Medical University of Plovdiv \\ ${ }^{2}$ Department of Health management, health economics and primary care, \\ Faculty of Public Health, Medical University of Plovdiv \\ ${ }^{3}$ Medical College, Medical University of Plovdiv
}

\begin{abstract}
INTRODUCTION: Various neighbourhood features such as safety, green spaces, aesthetics and maintenance of the built environment, etc. are correlates of health-related behaviours and outcomes. The assessment of the neighbourhood quality by raters, using audit tools, is an objective way to obtain reliable information about those features, and the Irvine-Minnesota Inventory (IMI) is one of the most widely used tools.

AIM: The aim of this study was to adapt IMI for Bulgarian settings by restructuring and modifying its dimensions, and to test the reliability of a desk-based (GIS) against a field audit version.

MATERIAL AND METHODS: IMI was adapted for Bulgarian settings (BVIMI). Then 180 street segments were selected from the city of Plovdiv, based on their characteristics, and 25\% were sampled to be audited. Three raters carried out the neighbourhood audits using a field version (in-person audits) and a GIS version of BVIMI. The inter- and intra-rater agreement, as well as the agreement between the GIS and field audits were tested with Krippendorff's alpha and the Intraclass correlation coefficient.

RESULTS: BVIMI has 86 items organised into six domains - "Safety", "Traffic", "Maintenance", "Nature and Landscape", "Attractiveness of the Build Environment" and "Types of Buildings and Land use". It is temporally stable and has high inter- and intra-rater agreement, except for some items necessitating subjective judgments (i.e. features and attitudes) or assessing unstable features (i.e. stray dogs).

CONCLUSIONS: BVIMI is a reliable GIS-based neighbourhood auditing tool adapted for Bulgarian urban settings. It might be routinely utilised by environmental hygienists, social epidemiologists and other public health experts to study the associations between various neighbourhood features and health behaviours/ outcomes.
\end{abstract}

Keywords: Irvine-Minnesota Inventory, GIS, neighbourhood audit, medical geography, environmental medicine, epidemiology

\author{
Address for correspondence: \\ Angel Dzhambov \\ Dept. Hygiene and ecomedicine, Faculty of Public Health, \\ Medical University of Plovdiv, \\ $15 A$ Vasil Aprilov Blvd., \\ 4002 Plovdiv, Bulgaria, \\ e-mail:angelleloti@gmail.com
}

Received: November 12, 2015

Accepted: March 9, 2016

\section{INTRODUCTION}

As people are influenced by their living environment, regardless of whether they perceive themselves as conditioned by it (1), the interest of public health community in urban planning, social epidemiology, medical geography and all fields related is rising. According to Macintyre et al. (2), in order to improve public health, the scientific research should be directed towards the people's living environment, 
Angel Dzhambov, Donka Dimitrova, Mariana Aleksandrova

its characteristics and its role in shaping human health. Another reason for this increasing interest is the fact that purely person-centred approaches overlook some important determinants of health (3). On the other hand, modifying urban environments is possible when local authorities and the government are involved (4), which may ultimately result in improving the health status of the population and reducing additional expenses for healthcare.

Health depends on both natural and built living environment (5). Mounting evidence suggests the value of neighbourhood quality for preventing socially significant diseases. Low neighbourhood quality is associated with decreased quality of life and fear (6, $7)$, low self-esteem and moral $(2,8)$ and poorer selfrated health (9). Conversely, abundance and diversity of residential greenness, for example, has been related to decreased mortality and morbidity $(10,11,12)$.

Walking behaviour is arguably the most prominent and thoroughly investigated health correlate of neighbourhood quality. The modern world is characterised by an "obesity pandemic" (13). Regular physical activity, on the other hand, may serve as a protective factor against weight gain and obesity, type 2 diabetes, cardiovascular and musculoskeletal disorders, amongst others $(14,15)$. However, there is inconsistency among different studies on environmental determinants of physical activity, which makes it difficult to draw strong conclusions. It has been hypothesized that the discrepancies are due to the mode of measurement of neighbourhood features (16). For children and adolescents, for example, the most consistent associations were found when the authors used objectively measured environmental features (16). Operating with a measure independent from the respondents is essential, because some people tend to overestimate the quality of their living environment (17) or they simply misclassify it in other systematic way; common method bias is also an issue when both the predictor and outcome variables are self-reported by the participants in the same context (18). Therefore, data obtained from assessment by independent trained raters are considered more reliable.

Various audit tools have been developed over the years to answer the call for a quantitative measure of neighbourhood features (19). The IrvineMinnesota Inventory (IMI) is among the most wide- ly applied (20). It has been used to study walking behaviour of students, school children and other residents $(20,21,22)$. Werner et al. (20) chose to adapt the IMI in France because audit tools provide somewhat objective collection of environmental data. It is also regarded both as very general and in the same time detailed. Schopflocher et al. (23) were interested in IMI as well, because it might be applied as the most comprehensive community audit tool. In the development (24) and reliability testing of IMI (25), however, the authors did not provide specific scoring instructions on how to reduce or aggregate the large amount of information collected by the 178 individual items. Boanet et al. (26) adopted a very unpractical approach to study the criterion validity of IMI they ran separate regressions with each item as a predictor of physical activity and proposed combining these items into scales, which turned out to be significant predictors. In fact, this is not only unpractical, but also with the number of tests, some statistical concerns arise as well. Werner et al. (20), on the other hand, reorganised some items in order for them to pertain only to a single domain and then they calculated standard scores for each item and aggregated them into scales representing the six domains. Recently, Schopflocher et al. (23) failed to support the viability of the scoring methodologies proposed by Boanet et al. (26) and Werner et al. (20). They expressed concerns about the statistical inference in previous research on IMI and concluded that it was difficult to establish the relationships between the features of the built environment and health-related behaviours using IMI. Thus, they proposed using some psychometrical approaches in order to provide adequate scoring protocol.

Neither IMI nor any other neighbourhood audit tool has been made available for academic use in Bulgaria. The aim of this study was to adapt IMI for Bulgarian settings by restructuring and modifying its dimensions, and then to test the inter-rater and intra-rater reliability of the Bulgarian Version of IMI (BVIMI), to test the reliability of a desk-based measure when compared with a field audit, and to examine the internal consistency of BVIMI. 


\section{MATERIAL AND METHODS}

\section{Conceptualisation of BVIMI}

The first step towards modifying IMI was for three raters to go through the original training protocol. As outlined by Day et al. $(24,27)$, IMI can be used by people who lack specific training or qualification in urban planning and design, such as college students and community members, as long as they have passed through the training protocol. For this reason our team comprised, on one hand, individuals involved in this research field, and, on the other, a layperson. One of the raters was appointed team leader, as he conceptualised the study. The raters first read the codebook describing the basic philosophy of IMI and giving detailed instructions regarding the individual items and their interpretation (27). Then they studied the PowerPoint training presentation (28). The training took up to five hours spread over two weeks.

After the initial introduction to the original IMI, we decided to modify it. We had several key objectives: (1) to adapt IMI given Bulgarian neighbourhoods' design and features; (2) to restructure it in order to facilitate further practical use of BVIMI for several intended projects; and (3) to test the agreement between a GIS-based and on-site auditing. Additional benefit would be to make IMI shorter and more practical; however, this was not a main objective. The operationalization of BVIMI was based on a literature review and the expert opinion of the authors. (a structured version of BVIMI is available at https://drive.google.com/ file/d/0B1DeADGAyPjnVWhfUVRhbXV0UWc/ edit?usp=sharing.) BVIMI comprises 86 items and five domains of neighbourhood quality - "Safety", "Traffic", "Maintenance", "Nature and Landscape", "Attractiveness of the Built Environment" and "Types of Buildings and Land Use". Only 14 new items were conceptualised and integrated in the original IMI. The rest were present in the original IMI exactly as is, or were modified - some items were fused (for example, the presence of civic and recreational facilities or the different retail types), while the scoring of others was altered in order for the items and domains to be positively coded. Another benefit of the revised scoring was that it provides a finer scale of measurement. Most of the new items were added in the domains "Nature and Landscape", which focuses on greenspace quality and characteristics, and several items were added to the "Safety" domain such as the presence of "bad dog" signs or video surveillance. The greenspace items were partly adopted from Edwards et al. (29). Necessary adjustments were made given the characteristics of Bulgarian urban settings. Some items were eliminated, because, for example, Bulgarian houses generally do not have front porches and the neighbourhoods rarely have identification signs. Finally, the street crossing features were included only as general questions regarding all the crosswalks on the segment (with new scoring system to account for the fusion of the items regarding all crosswalks along the segment) including mid-block crosswalks. The raters recorded summary features of both sides of the segment (items with adjusted scoring). All these changes were necessary because, it being the first reliable audit tool in Bulgaria, we wanted to extend the application of BVIMI beyond prediction of walking behaviour, and thus the relative weight of the crosswalk items was redistributed away.

\section{Study Area}

The city of Plovdiv is the second largest city in Bulgaria with a population of 339077 inhabitants and a territory of $101.98 \mathrm{~km}^{2}$. Plovdiv is divided into six administrative districts and possesses diverse urban settings and landscapes. It has a well-defined core with high integration to the South-West. The centre of the city is characterised by an old and dense street grid represented by small winding streets and inner-neighbourhood intersections, historic buildings, single-family homes, pedestrianised main street and diverse retail types of stores. The urban fabric in the outer concentric rings of the city is more coarse-grained with large-scale weaves, residential blocks and lower density of the built environment. The overall quality and maintenance are also higher in the finer-grained centre of Plovdiv. Several highly integrated boulevards stretch through the city and outwards. There are some spatially isolated areas close to the agricultural fields in Plovdiv's surroundings, the Roma ghetto and the old part in the city centre. All these characteristics allow the researcher to select segments sufficiently different on almost all neighbourhood quality features, in order to capture greater variance with BVIMI. 
Angel Dzhambov, Donka Dimitrova, Mariana Aleksandrova

\section{Audit Protocol}

We sampled 180 street segments in order to have diverse neighbourhood features represented in the sample. The sampling was guided primarily by the features that each segment represented, rather than by proportionality in the geospatial distribution of the segments. From those, a random sample of 45 segments (25\%) was selected (30). The length constraints for the segments were approximately $50-300 \mathrm{~m}$ according to Griew et al. (31). In order to facilitate data collection and to include segments with considerably varying geomorphological and planning characteristics, the tested units of reliability were segments rather than settings. Several debriefing sessions were carried out among the raters to achieve uniformity in the performance of evaluation. In addition, they were instructed to rely only on the available evidence when carrying out the audits disregarding any previous personal experiences, expectations or intimate knowledge of the segments.

At the first stage of data collection the raters did a virtual walk along the segments and documented observed characteristics using a printed paper version of BVIMI (the items in this version were organised so that they would facilitate and expedite the auditing procedure). We used Google Street View images for the city of Plovdiv dated from 2012 (March - June), which is about two years before the field observations took place in 2014. The results from a GIS version of BVIMI were compared to direct field observations, because in-person audits are time-consuming, costly, limit the scale of data collection and occasionally might pose a threat to the raters' safety (31); thus a GIS version of the instrument would be of practical value. Moreover, the main argument of the developers of IMI against GIS-only auditing was that "many built environment features that are potentially linked to active living have not yet been incorporated into local GIS databases (e.g., street trees, sidewalks)" (24). Google Street View displays video stills of streets in order to provide continuous panoramic street views that can be navigated along and rotated, allowing a virtual 3D walk (31). It offers $360^{\circ}$ horizontal and $290^{\circ}$ vertical panoramic views at the street level at height of about $2.5 \mathrm{~m}$ (32). Previously other authors have successfully attempted to validate similar urban audit tools as "desk tools" $(29,31,32,33,34)$. Due to the limitation of GIS, how- ever, some items in the original IMI (for example, "Is the dominant smell unpleasant?") were not included in BVIMI. In a couple of weeks' time the same segments were re-audited as a retest study in order to determine the intra-rater agreement of BVIMI. The GIS assessment procedures were carried out during the winter of 2013/2014 because Street View images are not conditioned on the season when they are being used. (In 2015 they were repeated to check for consistency by a fourth rater without prior training.)

During the spring of 2014 (about a month and a half later) the raters started visiting the previously audited street segments and filed a paper version of BVIMI recording their rating for the different residential quality features in order to measure the agreement between the GIS and field audits. The observers conducted independent, in-person observations, walking along each segment. The team leader instructed the other raters to replicate their virtual walk starting from the same end of the street and to try and capture as much relevant detail as possible even if this was not possible with Google Street View.

\section{Data Analytic Strategy}

Basic descriptive statistics, Pearson zero-order (on domain level) and Spearman (on individual item level) correlations were performed to study the internal relationships between the domains of BVIMI. The six domains of BVIMI were not multivariate normal (VQ3 $\left.{ }_{(12)}=110.61, \mathrm{p}<0.001\right)$. No outliers were detected.

Unlike all previous studies on the topic, we used Krippendorff's alpha coefficient $\left(\alpha_{\mathrm{K}}\right)$ (specifications: ordinal data) to determine the inter-rater agreement as well as the agreement between the GIS and field audits, and the intra-class correlation coefficient (ICC) (specifications: two-way mixed, absolute agreement) for the intra-rater reliability. The main reason for this choice was that, unlike Cohen's Kappa, alpha treats the raters as freely permutable and is not affected by their number, it renders the reliabilities for non-categorical data fully comparable across different metrics, and it also allows reliability data with missing categories (35). Finally, alpha adjusts itself for small sample sizes. Krippendorff's alpha defines reliability on a scale where 1.00 represents perfect reliability and 0.00 the absence of reliability. 
In order to examine the reliability of BVIMI, we applied several techniques: the internal consistency reliability of BVIMI was determined with Cronbach's alpha coefficient $\left(\alpha_{C}\right)$ and temporal stability via test-retest correlations. All analyses were carried out with SPSS v. 17.

\section{RESULTS}

According to Table 1, the raters generally had either perfect or very high agreement on almost all of the items. However, items 20 ("How safe do you feel walking on this segment?") and 62 ("How interesting is the architecture/urban design of this segment?") had low Krippendorff's alphas. After each rater evaluated the same segments in a couple of weeks, all items had ICC $\geq 0.93$ which averaged across ratersat, suggesting temporal stability of BVIMI. We report test-retest correlations $\geq 0.91$, for the team leader.
However, the most important step was to determine the degree of agreement between ratings based on a GIS virtual walk along the segments and real-life field observations. As evident from Table 1, only 21 items showed discrepancies; of those, the most problematic ones were items 17 ("How many "bad dog" signs are there on yard fences, doors, etc.?"), 20 ("How safe do you feel walking on this segment?"), 21 (“Are there any loose/unsupervised/barking dogs on this segment that seem menacing?") and 49 ("Are there flowers in the green space (if several, indicate overall)?"). Some of the items from "Maintenance" domain also yielded somewhat low agreement. It took approximately $6 \mathrm{~min}$. to complete the GIS version of BVIMI and about $13 \mathrm{~min}$. for the field version.

The next step was to determine the internal consistency of the domains. Except for "Mainte-

Table 1. Inter- and intra-rater agreement, descriptive statistics and reliability for the Bulgarian Version of Irvine-Minnesota Inventory

\begin{tabular}{|c|c|c|c|c|c|c|c|c|c|c|c|}
\hline \multirow[t]{3}{*}{ Domain } & \multicolumn{3}{|l|}{ Item } & \multicolumn{3}{|c|}{ Agreement } & \multicolumn{3}{|c|}{ Descriptives } & \multicolumn{2}{|c|}{ Reliability } \\
\hline & & \multicolumn{2}{|c|}{ GIS study } & \multirow{2}{*}{$\begin{array}{c}\begin{array}{c}\text { Field } \\
\text { study }\end{array} \\
a_{\mathrm{K}}\end{array}$} & \multirow{2}{*}{$\begin{array}{c}\text { GIS vs. Field } \\
\text { study } \\
a_{\mathrm{K}}\end{array}$} & \multirow[t]{2}{*}{ Min } & \multirow[t]{2}{*}{$\operatorname{Max}$} & \multirow[t]{2}{*}{ M } & \multirow[t]{2}{*}{ SD } & \multirow[t]{2}{*}{$a_{C}$} & \multirow{2}{*}{$\begin{array}{l}\text { Test- } \\
\text { retest }\end{array}$} \\
\hline & & $\alpha_{K}$ & ICC & & & & & & & & \\
\hline \multirow[t]{10}{*}{ Safety } & & & & & & 16 & 50 & 24.42 & 6.14 & .83 & \\
\hline & 9 & .72 & $\mathrm{n} / \mathrm{a}$ & .57 & .82 & 0 & 3 & 1.64 & .65 & $\mathrm{n} / \mathrm{a}$ & 1.00 \\
\hline & 10 & .80 & $\mathrm{n} / \mathrm{a}$ & .80 & $\mathrm{n} / \mathrm{a}$ & 0 & 3 & 1.56 & .78 & $\mathrm{n} / \mathrm{a}$ & 1.00 \\
\hline & 12 & .86 & $\mathrm{n} / \mathrm{a}$ & .86 & $\mathrm{n} / \mathrm{a}$ & 2 & 3 & 2.04 & .21 & $\mathrm{n} / \mathrm{a}$ & 1.00 \\
\hline & 14 & .96 & $\mathrm{n} / \mathrm{a}$ & .75 & .77 & 1 & 4 & 1.93 & .65 & $\mathrm{n} / \mathrm{a}$ & 1.00 \\
\hline & 15 & .99 & $\mathrm{n} / \mathrm{a}$ & .99 & $\mathrm{n} / \mathrm{a}$ & 0 & 2 & 1.76 & .48 & $\mathrm{n} / \mathrm{a}$ & 1.00 \\
\hline & 17 & $\mathrm{n} / \mathrm{a}$ & .93 & 1.00 & .48 & 1 & 2 & 1.98 & .15 & $\mathrm{n} / \mathrm{a}$ & 1.00 \\
\hline & 18 & $\mathrm{n} / \mathrm{a}$ & .99 & .96 & .93 & 0 & 2 & 1.42 & .72 & $\mathrm{n} / \mathrm{a}$ & 1.00 \\
\hline & 20 & .49 & $\mathrm{n} / \mathrm{a}$ & .17 & .56 & 0 & 2 & 1.76 & .57 & $\mathrm{n} / \mathrm{a}$ & 1.00 \\
\hline & 21 & $\mathrm{n} / \mathrm{a}$ & .98 & .13 & .03 & 0 & 2 & 1.91 & .36 & $\mathrm{n} / \mathrm{a}$ & .99 \\
\hline \multirow[t]{3}{*}{ Traffic } & & & & & & 0 & 34 & 27.98 & 6.52 & .87 & \\
\hline & 29 & $\mathrm{n} / \mathrm{a}$ & .98 & .75 & .81 & 0 & 3 & 2.16 & .74 & $\mathrm{n} / \mathrm{a}$ & .97 \\
\hline & 35 & .96 & .98 & .77 & .90 & 0 & 3 & 1.91 & .87 & $\mathrm{n} / \mathrm{a}$ & .91 \\
\hline \multirow[t]{4}{*}{ Maintenance } & & & & & & 2 & 11 & 7.69 & 2.10 & .49 & \\
\hline & 37 & .60 & $\mathrm{n} / \mathrm{a}$ & .74 & .69 & 1 & 3 & 2.09 & .60 & $\mathrm{n} / \mathrm{a}$ & 1.00 \\
\hline & 38 & $\mathrm{n} / \mathrm{a}$ & $\mathrm{n} / \mathrm{a}$ & .81 & .64 & 0 & 2 & 1.51 & .84 & $\mathrm{n} / \mathrm{a}$ & 1.00 \\
\hline & 39 & $\mathrm{n} / \mathrm{a}$ & .98 & .95 & .62 & 0 & 2 & 1.00 & .48 & $\mathrm{n} / \mathrm{a}$ & 1.00 \\
\hline
\end{tabular}


Angel Dzhambov, Donka Dimitrova, Mariana Aleksandrova

\begin{tabular}{|c|c|c|c|c|c|c|c|c|c|c|c|}
\hline & 40 & $\mathrm{n} / \mathrm{a}$ & .99 & .66 & .65 & 0 & 2 & 1.62 & .65 & $\mathrm{n} / \mathrm{a}$ & 1.00 \\
\hline & 41 & $\mathrm{n} / \mathrm{a}$ & $\mathrm{n} / \mathrm{a}$ & 1.00 & .84 & 0 & 2 & .93 & .69 & $\mathrm{n} / \mathrm{a}$ & 1.00 \\
\hline & 42 & $\mathrm{n} / \mathrm{a}$ & .97 & .93 & .81 & 0 & 2 & .53 & .66 & $\mathrm{n} / \mathrm{a}$ & .93 \\
\hline \multirow{12}{*}{$\begin{array}{l}\text { Nature And } \\
\text { Landscape }\end{array}$} & & & & & & 3 & 25 & 10.60 & 7.20 & .88 & \\
\hline & 43 & .92 & $\mathrm{n} / \mathrm{a}$ & .92 & $\mathrm{n} / \mathrm{a}$ & 0 & 2 & .60 & .62 & $\mathrm{n} / \mathrm{a}$ & 1.00 \\
\hline & 44 & .95 & .99 & .95 & $\mathrm{n} / \mathrm{a}$ & 0 & 2 & .87 & .89 & $\mathrm{n} / \mathrm{a}$ & 1.00 \\
\hline & 45 & .94 & $\mathrm{n} / \mathrm{a}$ & .94 & $\mathrm{n} / \mathrm{a}$ & 0 & 1 & .47 & .50 & $\mathrm{n} / \mathrm{a}$ & 1.00 \\
\hline & 46 & .95 & $\mathrm{n} / \mathrm{a}$ & .95 & $\mathrm{n} / \mathrm{a}$ & 0 & 3 & 1.09 & 1.2 & $\mathrm{n} / \mathrm{a}$ & 1.00 \\
\hline & 47 & .94 & $\mathrm{n} / \mathrm{a}$ & .94 & $\mathrm{n} / \mathrm{a}$ & 0 & 1 & .44 & .50 & $\mathrm{n} / \mathrm{a}$ & 1.00 \\
\hline & 48 & .94 & $\mathrm{n} / \mathrm{a}$ & .94 & $\mathrm{n} / \mathrm{a}$ & 0 & 1 & .49 & .51 & $\mathrm{n} / \mathrm{a}$ & 1.00 \\
\hline & 49 & $\mathrm{n} / \mathrm{a}$ & $\mathrm{n} / \mathrm{a}$ & 1.00 & .25 & 0 & 1 & .07 & .25 & $\mathrm{n} / \mathrm{a}$ & 1.00 \\
\hline & 50 & .88 & .97 & .87 & .71 & 0 & 3 & .67 & 1.09 & $\mathrm{n} / \mathrm{a}$ & 1.00 \\
\hline & 52 & .95 & .98 & .96 & .82 & 0 & 3 & .69 & 1.02 & $\mathrm{n} / \mathrm{a}$ & .99 \\
\hline & 53 & .95 & .99 & .95 & .96 & 0 & 3 & 1.02 & 1.18 & $\mathrm{n} / \mathrm{a}$ & .99 \\
\hline & 59 & .91 & $\mathrm{n} / \mathrm{a}$ & .89 & .87 & 0 & 2 & 1.29 & .63 & $\mathrm{n} / \mathrm{a}$ & 1.00 \\
\hline \multirow[t]{4}{*}{$\begin{array}{l}\text { Attractivenes } \\
\text { Build Enviror }\end{array}$} & $\begin{array}{l}\text { the } \\
\text { ent }\end{array}$ & & & & & 0 & 8 & 2.40 & 1.98 & .77 & \\
\hline & 60 & .66 & .98 & .66 & $\mathrm{n} / \mathrm{a}$ & 0 & 2 & 1.02 & .54 & $\mathrm{n} / \mathrm{a}$ & .96 \\
\hline & 62 & .52 & $\mathrm{n} / \mathrm{a}$ & .52 & $\mathrm{n} / \mathrm{a}$ & 0 & 2 & .49 & .66 & $\mathrm{n} / \mathrm{a}$ & 1.00 \\
\hline & 64 & .65 & $\mathrm{n} / \mathrm{a}$ & .65 & $\mathrm{n} / \mathrm{a}$ & 0 & 2 & .09 & .36 & $\mathrm{n} / \mathrm{a}$ & 1.00 \\
\hline \multirow[t]{5}{*}{$\begin{array}{l}\text { Types Of Buil } \\
\text { And Landuse }\end{array}$} & & & & & & 6 & 22 & 12.49 & 4.37 & .60 & \\
\hline & 66 & .95 & $\mathrm{n} / \mathrm{a}$ & .95 & $\mathrm{n} / \mathrm{a}$ & 0 & 2 & 1.18 & .78 & $\mathrm{n} / \mathrm{a}$ & 1.00 \\
\hline & 69 & .91 & $\mathrm{n} / \mathrm{a}$ & .91 & $\mathrm{n} / \mathrm{a}$ & 0 & 2 & .44 & .59 & $\mathrm{n} / \mathrm{a}$ & 1.00 \\
\hline & 71 & $\mathrm{n} / \mathrm{a}$ & $\mathrm{n} / \mathrm{a}$ & .79 & .76 & 0 & 1 & .07 & .25 & $\mathrm{n} / \mathrm{a}$ & 1.00 \\
\hline & 81 & $\mathrm{n} / \mathrm{a}$ & $\mathrm{n} / \mathrm{a}$ & .99 & .96 & 0 & 2 & 1.04 & .80 & $\mathrm{n} / \mathrm{a}$ & 1.00 \\
\hline
\end{tabular}

Note: Items with perfect agreement are omitted from the table; Reliability statistics are reported only for the composite scores of the domains; the authors agreed on reporting representative estimates for the scoring of rater A. D. (min, max, M, SD); GIS study: Inter-rater (Krippendorff's alpha) and intra-rater agreement (ICC, Intraclass correlation coefficient); Field test: Inter-rater agreement (Krippendorff's alpha); GIS vs. Field study: intra-rater agreement (Krippendorff's alpha); Reliability: M - mean, Min - minimum, Max - maximum, SD - standard deviation, Cronbach's alpha, Test-retest correlation; n/a - non-applicable; the ICC and Krippendorff's alpha for GIS $v$ s. Field study are reported as the mean estimate averaged from the three raters

nance" $\left(\alpha_{C}=0.49\right)$ and "Types of Buildings and Land Use" $\left(\alpha_{C}=0.60\right)$, all other domains had completely satisfactory alpha coefficients $\left(\alpha_{C} \geq 0.77\right)$.

We also report a correlation-matrix for the domains of BVIMI (See Table 2). Higher "Safety" scores were associated with less "Traffic", and higher scores on "Attractiveness of the Built Environ- ment" and "Types of Buildings and Land Use". On the other hand, "Traffic" and "Attractiveness of the Built Environment" were inversely correlated. The latter also had negative correlation with "Nature and Landscape". 
Table 2. Correlation-matrix for the Domains of the Bulgarian Version of Irvine-Minnesota Inventory

\begin{tabular}{lc|c|ccc} 
Domains & 1. & 2. & 3. & 4. & 5. \\
1. Safety & 1.00 & & & \\
2. Traffic & $-.76^{* *}$ & 1.00 & & & \\
3. Maintenance & .25 & -.19 & 1.00 & & \\
4. Nature and Landscape & -.15 & .24 & .13 & 1.00 \\
5. Attractiveness of the Build Environment & $.33^{*}$ & $-.53^{* *}$ & .23 & $-.32^{*}$ & 1.00 \\
6. Types of Buildings and Landuse & $.60^{* *}$ & -.26 & .06 & .29 & .12 \\
\hline
\end{tabular}

Note. $N=45$. Pearson zero-order correlation coefficients are reported. ${ }^{*}$ Correlation is significant at $p<0.05,{ }^{*}$ Correlation is significant at $p<0.01$

\section{DISCUSSION}

\section{Key Findings}

BVIMI has 86 items organised in six domains, representing some of the most commonly studied neighbourhood characteristics. It is significantly shorter than the original IMI and takes into account the characteristics of Bulgarian cities, which distinguish them from American, for which IMI was initially developed. BVIMI combines perceived safety from crime and traffic and operationalises "Traffic" as an independent domain because these are hypothesised to be able to impact on perceived neighbourhood acoustic environment (36). As already stated, we aimed at developing an audit tool, which would be applicable to different research scenarios, and not only to walking behaviour. "Maintenance", on its own, might be related to crime and incivilities $(6,7)$, while the presence and abundance of high quality and maintained green spaces and other natural elements might act as a protective measure and suppress such violent behaviours $(37,38)$. On its own, "Nature and Landscape" domain may be studied in reference to various psychosomatic health outcomes, as promoted by the PHENOTYPE study (39), including birth weight (40), psychological noise attenuation (41), etc. Finally, both attractiveness and diversity of the build environment can add to the value of a neighbourhood by increasing people's morale (7).

The reliability of BVIMI is satisfactory. Except for "Maintenance" and "Types of Buildings and Land Use" domains, all others have high internal consistency. A follow-up study might tackle this issue by redefining some of the items and their measurement. The inter-rater agreement in both the GIS and field studies were also very high; however, some items asking about perceived features and attitudes towards the street segment prove problematic due to the raters' individual differences. Regardless, given the high test-retest coefficients (ICC and correlations), the training protocol and the phrasing of the items seem to have been adequate, so that the ratings were not affected considerably by repeated measurements and temporal factors. It was reassuring to see that, when compared to the field observations, the GIS study yielded almost the same results on most of the items. Exceptions were some characteristics of the segment which can be particularly difficult to capture in time - namely, stray dogs are mobile and their presence on the segment is generally unpredictable, and the visual perspective that Google Street View offers is sometimes limited when it comes to evaluating green space features. Moreover, given that the raters were asked to disregard any previous knowledge of the segment and the fact that some of the images in the GIS study were taken during winter months, it was virtually impossible to determine whether, for example, flowers were present in the green space. As for "Maintenance" domain, the overall maintenance of Plovdiv has improved during the past couple of years and some construction sites have now been cleared, so it is not surprising that there is a somewhat lower agreement between the GIS and field audits.

Finally, it was revealed that due to the geomorphological features of the city and its planning, street segments with less traffic and more abundance, variability and pleasurableness of the build environment were rated as safer. On the other hand, traffic-heavy streets were rated as less attractive. It was controversial that more and higher quality residential green- 
Angel Dzhambov, Donka Dimitrova, Mariana Aleksandrova

ery was inversely related to safety, suggesting that some areas of the city might be too overbuilt, limiting the green space territory, which is a matter that might need to be addressed by local authorities; on the other hand, the positive (although non-insignificant) correlation between the presence of major boulevards and highways and greenery indicated that some of the consequences of traffic, such as air and noise pollutions, might be attenuated by the nearby green areas. The inverse correlation between "Attractiveness of the Built Environment" and "Nature and Landscape" suggests that there may be a disproportion in the quality and diversity of greenspaces (maintained by the Municipality) and the built environment

\section{Strengths and Limitations}

We wanted to construct easy-to-use tool for neighbourhood audits, which would be reliable in both GIS and field-observation scenarios depending on the needs for precision and resources of the researcher. Moreover, as medical geography is not sufficiently researched in Bulgaria, this paper would not only provide the necessary methodological tools to carry out applied research, but also underscores the important place that geographic, land-use and GIS techniques hold in modern public health research.

This was the first study to have attempted to adapt IMI in Bulgarian. BVIMI was constructed only for linear settings, but, if needed, it might easily be modified for nonlinear settings as well. To our knowledge, it was the first to compare field version of IMI with GIS desktop auditing version. The lack of detail in GIS software that Day et al. (24) argue about is not an issue nowadays when a Street View option is available. Google Street View is free service available in Google Earth. It was launched in 2007 (32), so this might explain the reservations that Day et al. (24) held towards GIS. A GIS version of IMI would reduce research resources and save valuable time on the cost of discrepancies in regards to some temporally unstable features. Because Bulgaria joined Google Street View relatively later than other countries, the images that we had at our disposal were from 2012. In other countries, longer time-gaps might yield slightly different reliability results, especially in quickly developing urban fabric.
We used Krippendorff's alpha coefficient to determine the degree of agreement between raters and between GIS and field observations, which is statistically superior technique to Cohen's Kappa or the percentage agreement among raters. The argument that Boarnet et al. (25) make to justify using the percent agreement (namely that "there was not enough variance in the data for kappa statistics to be a good measure of agreement") does not change the fact that the percent agreement is "easily calculated but flawed in nearly all important respects" (35).

Some items were re-scaled in order to provide a finer measurement scale. We also provided score calculation alternative by summing the individual item scores for the six domains. The latter were operationalised, based on theory and the relevant health outcomes that they could be related to. "Nature and Landscape" was especially improved by adopting some quality features from other green space audit tools.

However, this study has some noteworthy limitations. The items were organised into domains, based on educated guess, so further empirical validation of these constructs might be needed. (Researchers can modify BVIMI as they see fit.) Also, because of its reduced length and our attempt to capture important environmental correlates of various health behaviours and outcomes and not only walking, BVIMI represents a broader neighbourhood quality factor with scoring weights redistributed away from walkability features.

The agreement between the GIS and field ratings of some raters returned negative Krippendorff's alpha coefficients for items 20 and 21, which might indicate the existence of structural errors. These were items asking about perceived safety and the presence of stray dogs, which as mentioned above, are overly subjective items or depend on chance. The sample size was smallish (45 segments), but, on one hand, Krippendorff's alpha does not impose constraints on the sample size and on the other, it was comparable to that analysed by Griew et al. (31) and represented a $25 \%$ random sample from the initial 180 segments that we selected (30). Due to lack of funding, larger scale study was difficult to carry out. 


\section{Future Research}

Although we agree with Schopflocher et al. (23) that some psychometrical techniques might benefit further research on IMI and BVIMI respectively, there are several concerns that need to be discussed. First, it might be best to re-scale the items in order to generate a uniform multi-item scale, which will be easily fed to a factor analysis. On the other hand, the dogmatism of modern psychometrics has been challenged, when it comes to the underlying quantitative nature of psychological phenomena (42); given the fact that neighbourhood features tools cannot easily be classified as such (although they are conditioned by a collective social logic, a cognitive spatial idea, organising different building types, land use, green spaces, traffic flows, etc.), we are not convinced that using factor analysis might be theoretically feasible to uncover the dimensions of IMI. Given that such analysis is essentially based on correlations, and, not assuming a quantitative nature of the items, applying Bayesian methods and interpreting the items as ordered categories might partly solve the problem and provide further insight into the factorial structure of IMI. Moreover, approaches such as the Schmid-Leiman transformation might account for the fact that some items may load on several latent factors (for example, intensity of the traffic flow which can be interpreted as an element of both neighbourhood safety and environmental nuisance), by conceiving a set of second-order factors.

Essentially, the validity of BVIMI and its ability to adequately predict health-enhancing/damaging behaviours are much more relevant matters. Thus, apart from proving the predictive capacity of BVIMI, one might consider supplementing the walkability items with a special domain based on space syntax theory. Space syntax is an architectural technique which can predict human walking behaviour and navigation based on different structural and spatial organisation of the street network (43); that is, some street grids can foster active walking lifestyle, which emphasises the benefits of considering urban fabric for neighbourhood audit tools. However, not only could it be used as a complementary analysis along with BVIMI, but it could be incorporated in it by developing a set of items representing perceived spatial configuration of the segment (44).

\section{Implementation}

Neighbourhood environment, its spatial, physical and symbolic features can foster positive healthenhancing behaviours. However, the degree of social acceptance and political recognition by involved authorities that urban planning is not just architecture, but may serve as a health promotion platform, has not yet been reached in Bulgaria. Health promotion is the smart choice of all societies that for one reason or another have limited resources for healthcare (45). Unfortunately, Bulgarian researchers have been bereft of modern audit tools widely used worldwide to measure neighbourhood characteristics.

BVIMI might serve both practical and didactic roles. It will help to generate sufficient input data in order to create a database of neighbourhood scorings, which can then be used to study various healthrelated behaviours and the impact built environment has on health outcome variables. At the same time, initiatives such as this will help to expand the scope of environmental epidemiology in Bulgaria and its practical implications. Environmental hygienists, social epidemiologists and urban planners might routinely geocode and map various health indicators (not only prospectively, but retrospectively as well).

\section{CONCLUSIONS}

BVIMI is a reliable GIS-based neighbourhood auditing tool adapted for Bulgarian urban settings and the city of Plovdiv in particular. It is easy to use, it is cost-effective, showing high agreement between GIS and field observations. It might be used by environmental hygienists, social epidemiologists and other public health experts to study the associations between various neighbourhood features and health behaviours/outcomes. Future research should, however, re-evaluate BVIMI, its dimensions and scoring protocol in order to improve its performance. Applied research proving its criterion validity is highly warranted.

\section{REFERENCES}

1. Lim J. The moderating effects of vegetation on human violent behavior caused by environmental stressors. Thesis dissertation. The University of Texas at Arlington; 2005. Available from: https:// dspace.uta.edu/bitstream/handle/10106/30/umiuta-1193.pdf?sequence=1. Accessed March 5, 2014. 
Angel Dzhambov, Donka Dimitrova, Mariana Aleksandrova

2. Macintyre S, Maciver S, Sooman A. Area, class and health; should we be focusing on places or people? J Soc Pol 1993;22(02):213-34.

3. Diez-Roux AV. Neighborhoods and health: where are we and were do we go from here? Rev Epidemiol Sante Publique. 2007;55(1):13-21.

4. Dunstan F, Weaver N, Araya R, et al. An observation tool to assist with the assessment of urban residential. J Environ Psychol 2005;25(3):293-305.

5. Secretary's Advisory Committee on National Health Promotion and Disease Prevention Objectives for 2020. Healthy people 2020: An opportunity to address societal determinants of health in the U.S. Available from: http://healthypeople.gov/2020/ about/advisory/SocietalDeterminantsHealth.pdf. Accessed March 5, 2014.

6. Greenberg M. Improving neighbourhood quality: A hierarchy of needs. Hous Policy Debate 1999;10(3),601-24.

7. Cohen D, Spear M, Scribner R, Kissenger P, Mason $\mathrm{M}$, Wildgen J. "Broken Windows" and the risk of Gonorrhea. Am J Public Health 2000;90:230-6.

8. Forest R., Kearns A. Joined-up places? Social cohesion and neighbourhood regeneration. Joseph Rowntree Foundation, York; 1999.

9. Tomeya K, Diez-Roux AV. Clarkea P, Seemanc T. Associations between neighborhood characteristics and self-rated health: A cross-sectional investigation in the Multi-Ethnic Study of Atherosclerosis (MESA) cohort. Health Place 2013;24:267-74.

10. Takano T, Nakamura K, Watanabe M. Urban residential environments and senior citizens' longevity in megacity areas. The importance of walkable green spaces. J Epidemiol Community Health 2003;56(12):913-8.

11. Villeneuve PJ, Jerrett M, Su JG, et al. A cohort study relating urban green space with mortality in Ontario, Canada. Environ Res 2012;115:51-8.

12. Richardson E, Pearce J, Mitchell R, Day P, Kingham $\mathrm{S}$. The association between green space and cause-specific mortality in urban New Zealand: an ecological analysis of green space utility. BMC Public Health 2010;10:240.

13. Swinburn BA, Sacks G, Hall KD, et al. The global obesity pandemic: shaped by global drivers and local environments. Lancet 2011;378(9793):804-14.

14. Wang Y, Beydoun MA, Liang L, Caballero B, Kumanyika SK. Will all Americans become over- weight or obese? Estimating the progression and cost of the US obesity epidemic. Obesity (Silver Spring) 2008;16(10):2323-30.

15. Flegal KM, Carroll MD, Ogden CL, Curtin LR. Prevalence and trends in obesity among US adults, 1999-2008. JAMA 2010;303(3):235-41.

16. Ding D, Sallis JF, Kerr J, Lee S, Rosenberg DE. Neighborhood environment and physical activity among youth: A review. Am J Prev Med 2011;41:442-55.

17. Macintyre S, Ellaway A. Neighborhoods and health: An overview. In: Kawachi I, Berkman LF, eds. Neighborhoods and health. Oxford: Oxford University Press; 2003:20-42.

18. Podsakoff PM, MacKenzie SB, Lee JY, Podsakoff NP. Common method biases in behavioral research: a critical review of the literature and recommended remedies. J Appl Psychol 2003;88(5):879-903.

19. Nickelson J, Wang AR, Mitchell QP, Hendricks K, Paschal A. Inventory of the physical environment domains and subdomains measured by neighborhood audit tools: A systematic literature review. J Environ Psychol 2013;36:179-89.

20. Werner CN, Brown BB, Gallimore J. Light rail use is more likely on "walkable" blocks: Further support for using micro-level environmental audit measures. J Environ Psychol 2010;30:206-14.

21. Brown B, Werner C, Amburgey J, Szalay C. Walkability route perceptions and physical features, converging evidence for en route walking experiences. Environ Behav 2007;39(1):34-61.

22. Gallimore JM, Brown BB, Werner CM. Walking routes to school in new urban and suburban neighborhoods: An environmental walkability analysis of blocks and routes. J Environ Psychol 2011;31:184-91.

23. Schopflocher D, VanSpronsen E, Nykiforuk CI. Relating built environment to physical activity: two failures to validate. Int J Environ Res Public Health 2014;11(2):1233-49.

24. Day K, Boarnet M, Alfonzo M, Forsyth A. The Irvine-Minnesota inventory to measure built environments: development. Am J Prev Med 2006;30(2):144-52.

25. Boarnet MG, Day K, Alfonzo M, Forsyth A, Oakes $M$. The Irvine-Minnesota inventory to measure built environments: reliability tests. Am J Prev Med 2006;30(2):153-9. 
26. Boarnet MG, Forsyth A, Day K, Oakes JM. The street level built environment and physical activity and walking: Results of a predictive validity study for the Irvine Minnesota Inventory. Environ Behav 2011:43:735-75.

27. Day K, Boarnet M, Alfonzo M. Irvine Minnesota Inventory for observation of physical environment features linked to physical activity. Codebook; 2005. Available from: https://webfiles.uci.edu/kday/ public/index.html. Accessed November 27, 2014.

28. Alfonzo M, Day K, Boarnet M. Irvine Minnesota Inventory for observation of physical environment features linked to physical activity. Training protocol; 2005. Retrieved from: https://webfiles.uci. edu/kday/public/index.html. Accessed November 27, 2014.

29. Edwards N, Hooper P, Trapp GSA, Bull F, Boruff B, Giles-Corti B. Development of a Public Open Space Desktop Auditing Tool (POSDAT): A remote sensing approach. Appl Geogr 2013;38:22-30.

30. McMillan TE, Cubbin C, Parmenter B, Medina AV, Lee RE. Neighborhood sampling: how many streets must an auditor walk? Int J Behav Nutr Phys Act 2010;7:20. doi:10.1186/1479-5868-7-20

31. Griew P, Hillsdon M, Foster C, Coombes E, Jones A, Wilkinson P. Developing and testing a street audit tool using Google Street View to measure environmental supportiveness for physical activity. Int J Behav Nutr Phys Act 201323;10:103. doi: 10.1186/1479-5868-10-103.

32. Clarke P, Ailshire J, Melendez R, Bader MDM, Morenoff J. Using google earth to conduct a neighborhood audit: reliability of a virtual audit instrument. Health Place 2010;16(6):1224-9.

33. Rundle A, Bader MDM, Richards CA, Neckerman KM, Teitler JO. Using google street view to audit neighborhood environments. Am J Prev Med 2011;40(1):94-100.

34. Badland HM, Opit S, Witten K, Kearns RA, Mavoa S. Can virtual streetscape audits reliably replace physical streetscape audits. J Urban Health 2010:87(6):1007-16.

35. Hayes AF, Krippendorff K. Answering the call for a standard reliability measure for coding data. Communication Methods and Measures 2007;1(1):77-89.

36. Dzhambov A. Neighborhood quality as a modifier of environmental noise perception: bringing together environmental hygiene/medicine and en- vironmental psychology. Noise Health (submitted manuscript).

37. Kuo FE, Sullivan WC. Aggression and violence in the inner city: Effect of environment via mental fatigue. Environ Behav 2001;33:543-71.

38. Kuo FE, Sullivan W. Environment and Crime in the inner city: Does vegetation reduce crime? Environ Behav 2001;33:343-67.

39. Nieuwenhuijsen MJ, Kruize H, Gidlow C, et al. Positive health effects of the natural outdoor environment in typical populations in different regions in Europe (PHENOTYPE): a study programme protocol. BMJ Open 2014;4(4):e004951. doi: 10.1136/bmjopen-2014-004951.

40. Dzhambov A, Dimitrova D, Dimitrakova E. Association between residential greenness and birth weight: A systematic review and meta-analysis. Urban For Urban Gree 2014;13(4):621-29.

41. Dzhambov AM, Dimitrova DD. Urban green spaces' effectiveness as a psychological buffer for the negative health impact of noise pollution: A systematic review. Noise Health 2014;16(70):157-65.

42. Michell J. Normal Science, Pathological Science and Psychometrics. Theor Psychol 2000;10:639-67.

43. Hillier B. Space is the machine. Electronic edition. London: Space Syntax; 2007. Retrieved from: http://www.webcitation.org/6OMdMt09g. Accessed March 24, 2014.

44. Duncan MJ, Rashid M, Vandelanotte C, Cutumisu N, Plotnikoff RC. Development and reliability testing of a self-report instrument to measure the office layout as a correlate of occupational sitting. Int J Behav Nutr Phys Act 2013;10:16. doi: 10.1186/1479-5868-10-16.

45. World Health Organization, Constitution of the World Health Organization, 45th ed; 2006. Available from: http://www.who.int/governance/eb/ who_constitution_en.pdf. Accessed March 6, 2014. 